\title{
Mast cell chymase in pulmonary hypertension
} Yoshihide Mitani, Makiko Ueda, Kazuo Maruyama, Hideto Shimpo, Akiko Kojima,
Masahiko Matsumura, Kenzo Aoki, Minoru Sakurai

\begin{abstract}
Mast cells are known to be involved in various types of tissue remodelling but their role in pulmonary hypertension is still poorly understood. Two subtypes of mast cells have recently been identified by demonstrating one protease, chymase. This enzyme might be implicated in vascular remodelling. The changes in mast cells in lung tissue from three patients with primary or secondary pulmonary hypertension were therefore investigated. Compared with tissue from four control subjects the number of mast cells in the three patients was markedly increased, which suggests that chymase containing mast cells are involved in tissue remodelling accompanied by fibrotic changes in primary and secondary pulmonary hypertension.

(Thorax 1999;54:88-90)
\end{abstract}

Keywords: mast cells; pulmonary hypertension; chymase; tryptase

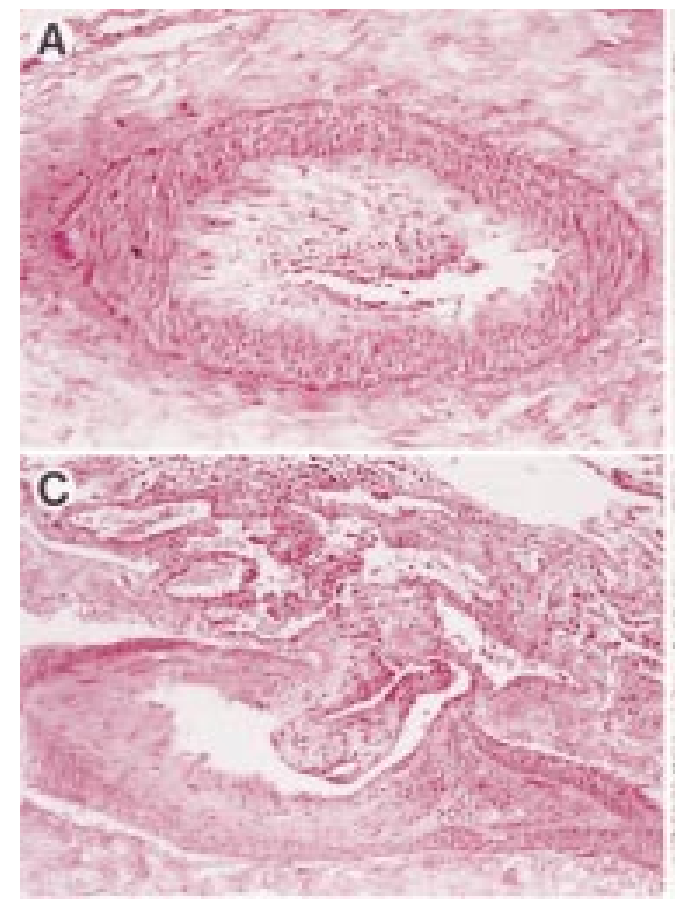

Early histochemical studies showed that the number of mast cells in the lung was increased in patients with primary and secondary pulmonary hypertension as well as in experimental models of pulmonary hypertension. ${ }^{12}$ However, the pathophysiological role of mast cells in pulmonary hypertension has not been fully elucidated. Mast cells are known to participate in various types of tissue remodelling through release of biologically active mediators including proteases (tryptase and chymase). ${ }^{3}$ Mast cells are composed of two phenotypes: mucosal mast cells which contain tryptase but not chymase, and connective tissue type mast cells which contain both proteases. ${ }^{3}$ However, the significance of the heterogeneity remains unknown. Recent studies have suggested that mast cell chymase converts angiotensin I to angiotensin II, suggesting the involvement of this protease in vascular remodelling after balloon injury. ${ }^{4}$ Since angiotensin II is involved in pulmonary hypertension and its vascular changes in chronically hypoxic rats, ${ }^{6}$ mast cell

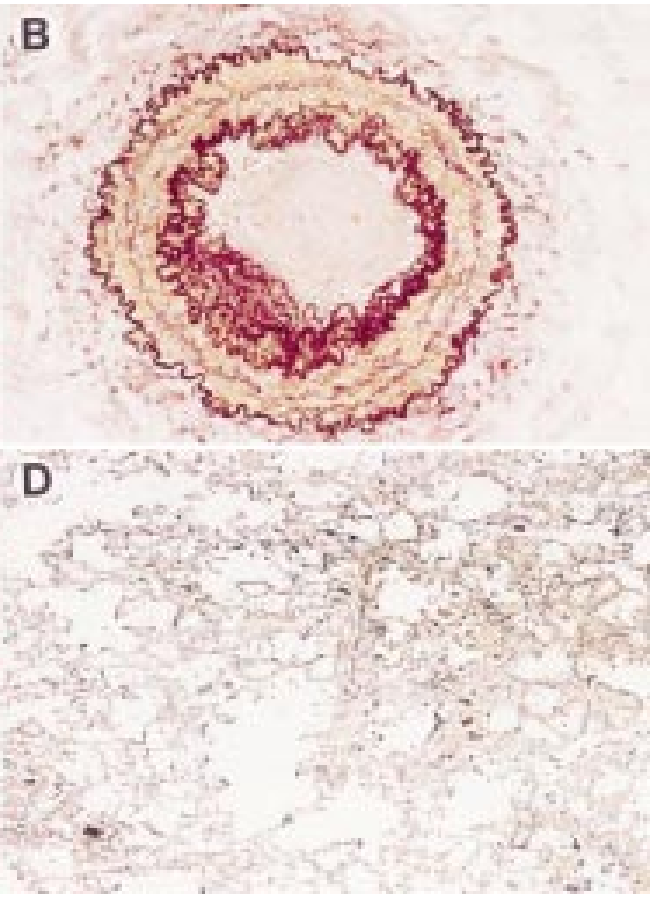

Figure 1 Lung sections showing representative vascular and parenchymal lesions in primary pulmonary hypertension: (A) cellular intimal thickening; (B) intimal fibrosis; (C) a plexiform lesion; and (D) a lesion with localised parenchymal fibrosis and mild septal fibrosis in patient 2. Stains: haematoxylin and eosin ( $A$ and $C$ ), Weigert's elastic van Gieson stain $(B$ and D). Original magnification: $A, \times 250 ; B, \times 350 ; C, \times 200 ; D, \times 40$. 


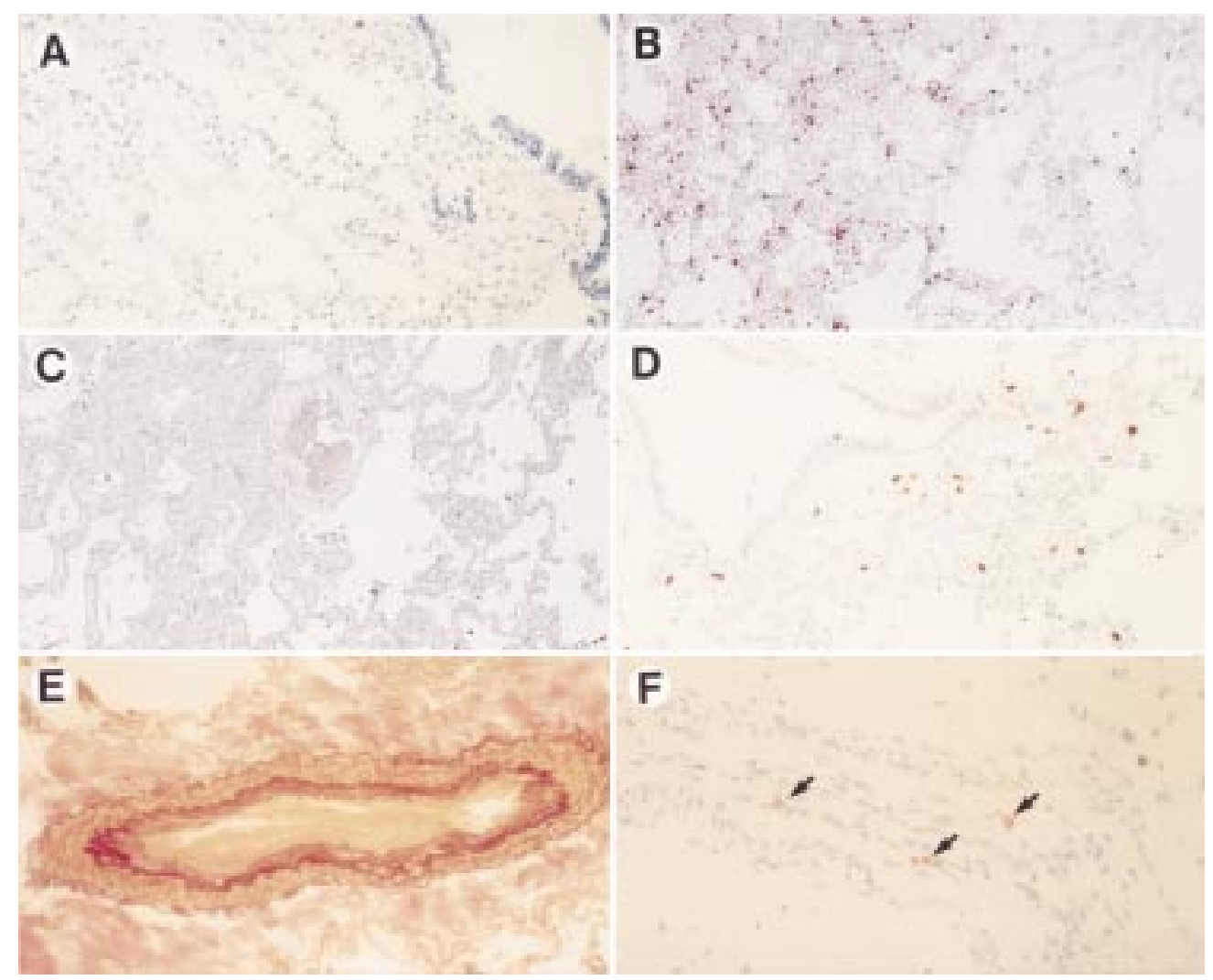

Figure 2 Immunostaining for chymase $(A, B, D$, and $F)$, negative control using a non-immune IgG antibody (C), and Weigert's elastic van Gieson stain (E). (A) Few chymase positive mast cells in a control subject; (B) numerous chymase positive mast cells in an area with interstitial fibrosis and neovascularisation in patient 2; (C) a negative control corresponding to patient in (B) showing absence of staining; (D) diffuse distribution of chymase positive mast cells in the lung parenchyma of patient $3 ;(E)$ and $(F)$ a vascular lesion with intimal fibrosis in patient 1 showing some chymase positive mast cells (arrows) within the intima. Original magnification: $A, \times 100 ; B, \times 60 ; C, \times 60 ; D, \times 100 ; E, \times 200 ; F$, $\times 250$.

chymase might contribute to these changes. In the present study we have analysed immunocytochemically lung tissues from patients with primary and secondary pulmonary hypertension and controls using specific antitryptase and antichymase antibodies.

\section{Case reports}

Lung tissue obtained at necropsy from two patients with primary pulmonary hypertension (patients 1 and 2) and a biopsy sample from a patient with pulmonary hypertension associated with patent ductus arteriosus (patient 3) were compared with samples of lung tissue from four control subjects (three men).

Patient 1 was an eight year old boy who was referred to our hospital because of an episode of fainting and oedema. He was in the terminal stage of the disease as indicated by cardiomegaly (cardiothoracic ratio 0.65 ) on chest radiography, and suprasystemic pulmonary arterial pressure on ultrasound examination. These findings, together with those of magnetic resonance imaging and lung perfusion scanning, revealed no other causes of pulmonary hypertension. He showed progressive heart failure regardless of medication and died six months later.

Patient 2 was a 28 year old man who had been fairly stable under observation for 10 years after an initial episode of haemoptysis. Cardiac catheterisation was performed seven months before he died of heart failure; the pulmonary to aortic systolic pressure ratio was 0.83 with no pulmonary vascular response to inhaled nitric oxide. No other causes of pulmonary hypertension were revealed.

Patient 3 had pulmonary hypertension associated with high pulmonary flow caused by patent ductus arteriosus, as indicated by equivalent pulmonary hypertension and a high pulmonary to systemic blood flow ratio (3.48) on preoperative cardiac catheterisation.

All of the control subjects had a relapsed malignant tumour but none had received anticancer chemotherapy within three months before death and all were free of neutropenia at the time of death. In each patient pathological examination confirmed the lack of metastatic tumours or inflammation in the lung.

The tissues were fixed in methanol Carnoy's fixative $(60 \%$ methanol, $30 \%$ chloroform, and $10 \%$ glacial acetic acid) and embedded in paraffin. Twenty serial sections of $5 \mu \mathrm{m}$ thickness were cut from each block. The first and second sections were stained with haematoxylin-eosin and Weigert's elastic van Gieson stain, respectively, and the other sections were used for immunocytochemical staining. Anti-human tryptase and chymase monoclonal antibodies (Cosmo Bio Co Ltd, Tokyo, Japan) were used and the labelled streptavidin-biotin complex system with peroxidase was employed for colour detection. Peroxidase activity was visu- 
alised with 3-amino-9-ethylcarbazole and sections were counterstained with haematoxylin. The lung sections were systematically scanned and the number of each group of mast cells per high power field $(\times 400)$ was counted in all fields of each section; the ratio of chymase positive to tryptase positive cells (C/T ratio) per high power field was calculated.

The characteristic cellular intimal thickening, intimal fibrosis, and plexiform lesions were found in patients 1 and 2 (Heath-Edwards grade 4 ; fig $1 \mathrm{~A}-\mathrm{C})$. Cellular intimal thickening contained cells that exhibited oval nuclei and copious cytoplasm with scant deposition of elastin, while intimal fibrosis showed dense deposition of elastin. In addition, in patient 2 localised fibrosis and mild septal fibrosis associated with neovascularisation were occasionally found (fig 1D). Patient 3 showed little medial thickening and no intimal lesions. In control patients there were no vascular or parenchymal lesions and only a few tryptase containing mast cells were found in the lung parenchyma (mean (SE) 3.3 (0.6) per high power field). Chymase positive mast cells were very rarely found (fig $2 \mathrm{~A}$ ), as shown by the $\mathrm{C} / \mathrm{T}$ ratio of $3.5(1.2) \%$. In patients 1 and 2 with primary pulmonary hypertension, mast cells were increased more than 10 -fold and were scattered diffusely in the lung parenchyma; the number of mast cells was 42.6 per high power field in patient 1 and 35.0 per high power field in patient 2 . In patient 3 mast cells were also diffusely increased in the lung parenchyma (13.9 per high power field). The $\mathrm{C} / \mathrm{T}$ ratio was increased in patients $2(53.0 \%)$ and $3(34.1 \%)$, but not in patient $1(2.0 \%)$ (fig $2 \mathrm{~B}$ and $\mathrm{D})$. The specificity and results obtained with antihuman tryptase and chymase antibodies were checked by omitting the primary antibodies and using a non-immune mouse IgG antibody (Dako, Glostrup, Denmark) as a negative control (fig 2C). Chymase positive mast cells were occasionally found in vascular lesions with intimal fibrosis but not with proliferative neointimal hyperplasia (fig $2 \mathrm{E}$ and $\mathrm{F}$ ). In patient 2, moreover, chymase positive mast cells were preferentially accumulated in the area of interstitial fibrosis admixed with newly formed microvessels (fig $2 \mathrm{~B}$ ).

\section{Discussion}

In this study we have investigated immunocytochemically lung tissues from patients with pulmonary hypertension using Carnoy's fixation and staining with antichymase and antitryptase monoclonal antibodies. The major advantages of our approach are that we can evaluate the phenotypic change in mast cells ${ }^{7}$ and this method is more sensitive in detecting mast cells than the conventional method using formalin fixation and staining granule proteoglycan with cationic dyes. ${ }^{78}$ The increase in tryptase positive mast cells in patients with pulmonary hypertension was consistent with the results of previous studies using the conventional methods. ${ }^{2}$ Initially we hypoth- esised that there may be a phenotypic change in mast cells in the lung from mucosal to connective tissue type in pulmonary hypertension. However, the phenotypic change was not always found in patients with pulmonary hypertension (patient 1), suggesting that mast cell chymase may not be essential to the increase in pulmonary arterial pressure.

In the present study, however, we found occasional chymase positive mast cells in vascular lesions with intimal fibrosis. This is of interest since the presence of chymase activity is associated with the intimal hyperplasia in balloon injured arteries. ${ }^{4}$ However, our histological findings did not suggest that mast cell chymase contributed to the formation of neointimal hyperplasia as these mast cells were detected only in the fibrous intima and not in proliferative neointimal lesions. These data raise the possibility that chymase positive mast cells might be implicated in the fibrous changes of the neointima. Furthermore, focal accumulation of chymase positive mast cells was found in the area with interstitial fibrosis and neovascularisation. These new findings suggest that chymase positive mast cells contribute to the tissue remodelling - that is, focal fibrosis and newly formed microvasculature-found in pulmonary hypertension via the mitogenicity of fibroblasts and the angiogenic activity, as well as proteolysis induced by the various mediators released from mast cells, as reported in pulmonary fibrosis and angiogenic lesions such as tumour invasion. ${ }^{9} 10$

In summary, our preliminary results suggest that chymase positive mast cells may contribute to tissue remodelling accompanied by fibrotic changes in primary pulmonary hypertension, rather than to the increase in vascular tone or formation of neointimal hyperplasia in pulmonary vessels. Future studies using the present method in a larger number of cases are required to elucidate further the role of mast cells in pulmonary hypertension.

1 Kay JM, Waymire JC, Grover RF. Lung mast cell hyperplasia and pulmonary histamine-forming capacity in hypoxic rats. Am $\mathcal{f}$ Physiol 1974;226:178-84.

2 Heath D, Yacoub M. Lung mast cells in plexogenic pulmonary arteriopathy. $\mathcal{F}$ Clin Pathol 1991;44:1003-6.

3 Galli SJ. New insights into the riddle of the mast cells: microenvironmental regulation of mast cell development and phenotypic heterogeneity. Lab Invest 1990;62:5-33.

4 Shiota N, Okunishi O, Fukamizu A, et al. Activation of two Shiota $\mathrm{N}$, Okunishi O, Fukamizu A, et al. Activation of two
angiotensin-generating systems in the balloon-injured angiotensin-generating systems in
artery. FEBS Lett 1993;323:239-42.

5 Urata H, Boehm KD, Philip A, et al. Cellular localization and regional distribution of an angiotensin II-forming chymase in the heart. $\mathcal{F}$ Clin Invest 1993;92:1269-81.

6 Morrell NW, Morris KG, Stenmark KR. Role of angiotensin-converting enzyme and angiotensin II in Physiol 1995;269(4 pt 2):H1186-94.

7 Kaartinen M, Penttila A, Kovanen PT. Mast cells of two types differing in neutral protease composition in the human aortic intima: demonstration of tryptase- and human aortic intima. demontration fatty streaks, and the shoulder region of atheromas. Arterioscler Thromb 1994:14:966-72

8 Shanahan F, Niven IM, Dyck N, et al. Human lung mast cells: distribution and abundance of histochemically distinct subpopulations. Int Arch Allergy Immunol 1987;83: distinct subp

9 Schulman ES. The role of mast cells in inflammatory responses in the lung. Crit Rev Immunol 1993;13:35-70.

10 Roche WR. The nature and significance of tumor-associated mast cells. $\mathcal{F}$ Pathol 1986;148:175-82. 